\title{
Modelling the diffusivity of mortar and concrete using a three- dimensional mesostructure with several aggregate shapes
}

\author{
S. Dehghanpoor Abyaneh *, H.S. Wong, and N.R. Buenfeld \\ Concrete Durability Group, Department of Civil and Environmental Engineering, Imperial College London
}

* Corresponding author. Tel.: +44 (0)20 75941195.

E-mail address: sdehghan@imperial.ac.uk (S. Dehghanpoor Abyaneh).

\begin{abstract}
This paper presents a numerical investigation into the effect of ITZ and aggregate shape on the diffusivity of mortar and concrete using a three-dimensional model. Concrete is treated as a three-phase composite consisting of aggregate particles, bulk cement paste and aggregate-paste interface, i.e. the 'interfacial transition zone' (ITZ). The model is set up in two stages. First, a three-dimensional representative volume element of the concrete mesostructure is generated. Then, a finite difference method is used to simulate molecular diffusion through the mesostructure. The transport properties of the conductive phases (bulk cement paste and ITZ) are determined based on the water/cement ratio, degree of hydration and porosity gradients away from aggregate particles. The model is validated against available experimental data and compared with analytical relationships for ideal cases. The model is then used to study the effect of aggregate shape on diffusivity, which has not been attempted before in three-dimensions. The model is also applied to assess the effects of water/cement ratio, degree of hydration, aggregate size, volume fraction, shape and orientation, ITZ width and percolation on diffusivity. Some of these effects are impractical to quantify from laboratory experimentation alone. It was found that the shape and orientation of aggregate particles have a significant effect on diffusivity. Diffusivity decreased when spherical aggregate particles are replaced with ellipsoidal particles due to the consequent increase in tortuosity of the cement paste.
\end{abstract}

Keywords: Concrete; Mortar; Cement-based materials; Modelling; Diffusivity; Aggregate shape

Highlights:

- Diffusivity of cementitious materials is modelled using a three-dimensional model.

- The required characteristics of Representative Elementary Volume were investigated.

- The simulations were verified by comparing with experimental and analytical results.

- The effects of different variables influencing diffusivity were investigated.

- The diffusivity decreased with the increase of aspect ratio of aggregate particles.

\section{Introduction}

Diffusion is one of the most important transport processes influencing the durability of cement-based materials. Therefore, the ability to estimate the diffusivity of concrete based on mixture proportions and microstructure is attractive, as it would assist in the development of service life prediction models and durability-based design codes.

Lab based transport testing has been carried out for many years to gain a better understanding of different variables influencing the diffusivity of cement-based materials. Since the microstructure of concrete is highly complex and its transport properties are influenced by many interacting parameters, many experiments should be carried out in order to identify the effect of different variables. However, it is often difficult to isolate the effects of specific variables because other influencing parameters inevitably vary. While some of these effects can be reduced or avoided entirely, others are difficult if not impossible to control, and so must be accounted for when analysing results. Moreover, experiments are time-consuming and expensive.

Thus, it would be extremely useful to be able to predict diffusivity based on the microstructure of concrete, either using an analytical or numerical model. However, existing analytical models are restricted to simplified microstructures, e.g. spherical aggregate particles dispersed in a medium [1, 2], so they are likely to be 
inaccurate. The tremendous increase in computational capabilities has strongly favoured the development of numerical simulations based on more precise description of the microstructure. Bentz et al. [3] used random walk simulations to study the diffusivity of concrete. Concrete is assumed to be a composite material consisting of aggregate particles, ITZ and bulk cement paste, and all three phases are treated as uniform continuum materials. Kamali-Bernard et al. $[4,5]$ computed the diffusivity of mortars from a digitized mesostructure by applying Fick's law. The mortar is assumed as a composite, in which the transport properties of the conductive phases (bulk cement paste and ITZ) are constants obtained by fitting experimental results. Zheng et al. [6] proposed a three-phase composite sphere model to calculate the steady-state chloride diffusivity of concrete. However, the simulations were carried out using a composite sphere model and may not be sufficient to capture the effects of ITZ percolation and tortuosity due to multisized aggregate particles.

Representing aggregate particles as spheres in a model is probably a gross simplification since the aggregate shape can affect the properties of concrete. Most modelling works published to date have been carried out assuming spherical aggregate particles. Recently, some researchers have attempted to model the influence of aggregate shape on the transport properties of cement-based materials. However, the simulations were carried out in two dimensions and the findings appear to be inconclusive $[7,8]$. Zheng at al. [7] used a two dimensional lattice model to investigate the effect of elliptical aggregate shapes on transport properties. They found that when the aspect ratio of elliptical aggregate particles increases, the chloride diffusivity in concrete decreases for a given chloride diffusivity ratio of ITZ to cement paste. Li et al. [8] used the finite element method to investigate chloride diffusion in concrete. The concrete is treated as a twodimensional material composed of only cement paste and aggregate particles. They found that the shape of aggregate has a small influence on the diffusion coefficient of chlorides in concrete. To the authors' knowledge, the effect of general aggregate shape on the transport properties of mortar and concrete has not been investigated in three-dimensions.

This paper presents a numerical investigation into factors influencing the diffusivity of mortars and concretes. This approach is particularly useful because a large number of related factors can potentially influence diffusivity. Many laboratory experiments would be required in order to understand their significance and interactions, and even so, may not be able to isolate all of these effects. For example, it is impossible to change the width of the ITZ and examine its effect on transport independent of other variables in a laboratory experiment. Increasing the volume fraction of ITZ (for example by changing the aggregate shape or particle size distribution) causes densification of the bulk cement paste and may change the tortuosity of the cement paste. These have different effects on transport properties, but cannot be isolated in experimental studies.

In order to investigate the effect of aggregate shape, aggregate particles are modelled as ellipsoids with varying aspect ratios, thus providing a dynamic range of shapes from spheres to tri-axial ellipsoidal, prolate and oblate spheroidal to resemble elongated or flaky aggregate shapes. The developed model is regarded as interactive because the transport properties of the ITZ and bulk paste are estimated from their porosities, which depend on the ITZ characteristics (width, porosity gradient), water/cement ratio and degree of hydration. The total porosity of the cement paste (ITZ + bulk paste) is set to be equal to the porosity of a cement paste of the same water to cement $(\mathrm{w} / \mathrm{c})$ ratio and hydration degree. A realistic aggregate size distribution is used as an input to the model. The model was validated against available theoretical and experimental results and was then applied to evaluate the effects of aggregate shape, content, size and orientation, water/cement ratio, degree of hydration, ITZ width and percolation on diffusivity.

\section{Approach}

To model transport phenomena in any material, an input structure coupled with a transport algorithm is required. Therefore, our approach consists of the following steps. First, a three-dimensional mesostructure is generated where concrete is idealised as a composite of aggregate particles, bulk cement paste and ITZ. An algorithm is utilised to convert the mesostructure into a corresponding mesh based on voxel analysis. The model may also include other phases such as air voids and cracks. The transport property of each phase is then defined according to its pore volume fraction. The ITZ is not assumed to be a single shell of uniform property, instead the porosity and diffusivity of the ITZ are allowed to vary with distance from the aggregate surface and with the progress of hydration. After a desired hydration degree is achieved, the capillary porosity is calculated as a function of distance from the nearest aggregate surface and is converted into diffusivity using a previously derived relationship [9]. Then the ITZ diffusivity is averaged over its thickness. A finite difference scheme is applied to the entire mesostructure to simulate bulk diffusion at steady-state conditions. This approach is advantageous because a fairly representative description of the microstructure is used as an input. Since the approach is applied to a digitised mesostructure, it serves as a platform for applying real images of microstructure as inputs in the future, and other transport properties such as 
pressure-induced water flow and capillary absorption of water can be simulated using the appropriate lattice methods. The finite difference technique requires less computational resource compared to finite element methods, thus investigation at higher discretisation is possible which may lead to improved accuracy.

\section{Generation of the model}

\subsection{Mesostructure}

To generate the mesostructure of a mortar or concrete, the aggregate size distribution is first determined either experimentally in a conventional sieve analysis or generated from a theoretical gradation such as Fuller-Thompson [10]. The smallest aggregate size considered was $0.15 \mathrm{~mm}$. Aggregate particles within each sieve class are substituted with identical spheres at the required volume fraction, and placed randomly in the computational cube with the largest particles first with no overlaps. A periodic boundary condition is applied to all sides of the computational cube. In doing so, aggregate particles that extend beyond the cube are completed (wrapped) into the opposite face of the cube. For a cube of $7.5 \mathrm{~mm}$ containing $30 \%$ volume of aggregate particles ranging from 0.15 to $2 \mathrm{~mm}$ (Fig .1), around eight thousand aggregate particles were required. Voxels representing the cement paste matrix are classified as either ITZ or bulk paste depending on their location relative to the nearest aggregate particle. A large number of voxels are required to realistically represent the mesostructure, but this comes at higher computational cost. A suitable sample size and resolution to obtain representative results will be examined later in this paper.

\subsection{Non-spherical aggregate particles}

Non-spherical aggregate particles are incorporated into the model to examine the effect of aggregate shape on transport properties. For this study, a range of ellipsoidal particles will be used. An ellipsoid is defined by the following equation:

$$
(x-\boldsymbol{v})^{T} A^{-1}(x-\boldsymbol{v})=1
$$

Where $v$ is a vector representing the center of the ellipsoid and $A$ is a positive definite matrix

with eigenvectors which are the principal directions of the ellipsoid and eigenvalues which are the squares of the semi-axes: $a^{2}, b^{2}$ and $c^{2}$.

The mesostructure is projected into a regular lattice, so collision and overlap detection is a straightforward exercise of finding out whether two particles occupy the same site(s), rather than having to compute and test intersections between any two particles, which is usually very expensive for non-spherical particles [11]. By varying the ratio $a: b: c$, four distinctive shapes ranging from spheres to elongated and disc shaped particles can be obtained. Table 1 shows the types of ellipsoids that will be used to represent aggregate particles in this study. Note that the ratios $a: b: c$ were selected such that the particle volume $(=4 / 3 \pi a b c)$ remains constant. Fig. 1 shows examples of randomly generated mesostructure containing $30 \%$ vol. of spherical and non-spherical aggregate particles. We note that this approach of projecting the microstructure into a regular lattice has already been applied at the pore-scale [12-14]. For example, Nabovati et al. [14] used this approach, coupled with the lattice Boltzmann method, to investigate the effect of curvature and aspect ratio of fibres on the permeability of fibrous porous media.

Table 1 Shapes used to represent aggregate particles

\begin{tabular}{lll}
\hline Shape & & $(a: b: c)$ \\
\hline Spherical & $(a=b=c)$ & $(1: 1: 1)$ \\
Tri-axial ellipsoidal & $(a>b>c)$ & $(2: 1: 0.5),(3: 1: 0.33)$ \\
Prolate spheroidal & $(a=b<c)$ & $(0.82: 0.82: 1.5),(0.71: 0.71: 2)$ \\
Oblate spheroidal & $(a=b>c)$ & $(1.5: 1.5: 0.44),(2: 2: 0.25)$ \\
\hline
\end{tabular}

\subsection{Diffusivity of the aggregate, ITZ and bulk cement paste}


The transport property of each component in the mesostructure is defined according to its porosity. Since most aggregates used in concrete are dense compared to the cement paste, we will assume that they have zero diffusivity in this study. Thus, mass transport occurs entirely through the interconnected cement paste matrix. The diffusivity of the cement paste depends on its capillary porosity, which in turn is a function of the $\mathrm{w} / \mathrm{c}$ ratio, degree of hydration and distance from the nearest aggregate surface.

Fig. 2 shows an example of the distribution of porosity in the cement paste for an OPC concrete with w/c ratio of 0.4 after 28 days curing, obtained by Crumbie [15] using image analysis on backscattered electron micrographs. Porosity is highest near the interface and decreases with distance from the aggregate surface. The width of the ITZ is typically about 25 to $50 \mu \mathrm{m}$, depending on the size of cement particles and bleeding effects. However, the shape of the porosity gradient appears to be independent of w/c ratio and degree of hydration, and so the porosity distribution shifts vertically depending on the total capillary porosity [15-18]. The porosity gradient can be approximated using the following exponential function:

$$
\phi(x)=\phi_{\text {bulk }}+0.2 \exp (-0.07 x)
$$

Where $\phi(x)$ is the capillary porosity at a distance $x$ from the nearest aggregate surface, and $\phi_{\text {bulk }}$ is the capillary porosity of the bulk cement paste $(=0.1$ for the data presented in Fig. 2$)$. We note that although the averaged ITZ porosity displays well-defined gradients, the local ITZ characteristic is heterogeneous and spatially variable [19]. Furthermore, the width of the ITZ is overestimated due to the random orientation by which the interfaces are measured in 2D image analysis [20].The true porosity gradient of the ITZ may be determined using 3D image analysis, or a combination of optical and backscattered electron imaging [21]. For the sake of simplicity, we will assume the porosity gradient around every aggregate particle in our mesostructure to be represented by Eq. 2 and no attempt is made to correct for the effects described above. As such, the influence of ITZ on transport properties may be exaggerated, but we will show later that this is a minor effect.

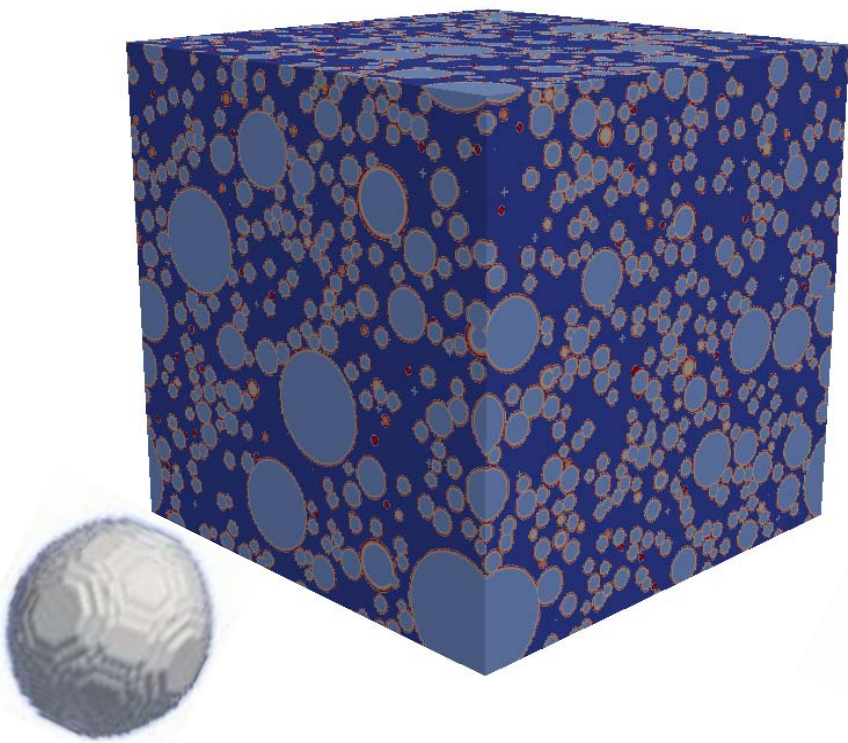

a) Spherical aggregate particles (a:b:c = 1:1:1)

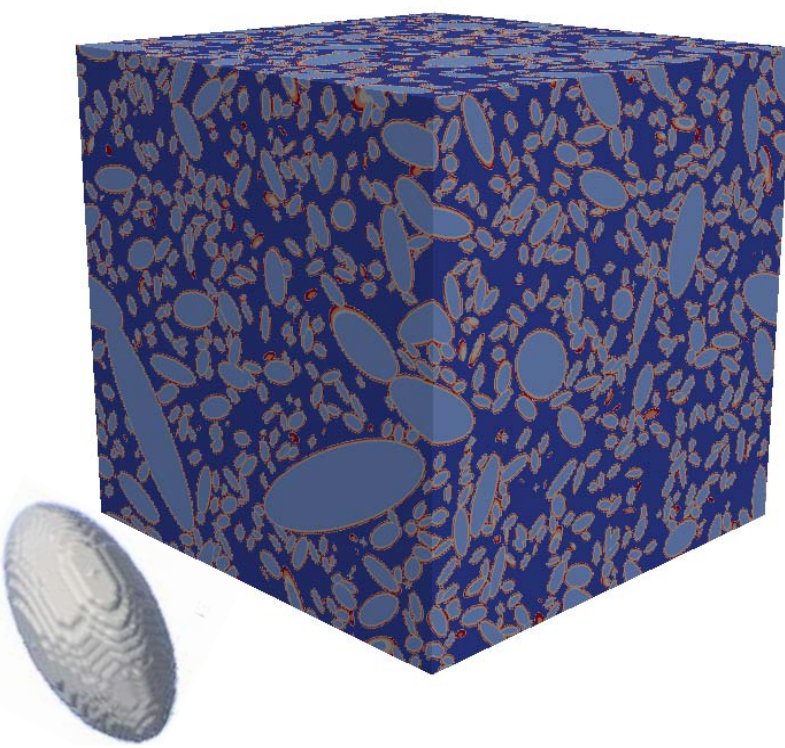

b) Tri-axial ellipsoidal aggregate particles (a:b:c $=2: 1: 0.5)$ 


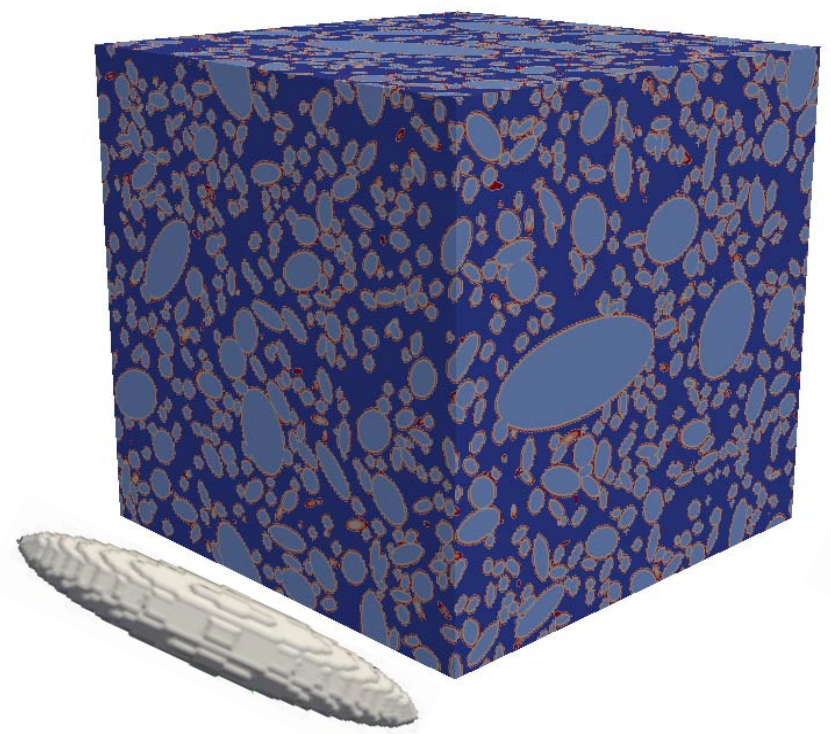

c) Prolate spheroidal aggregate particles $(a: b: c=0.71: 0.71: 2)$

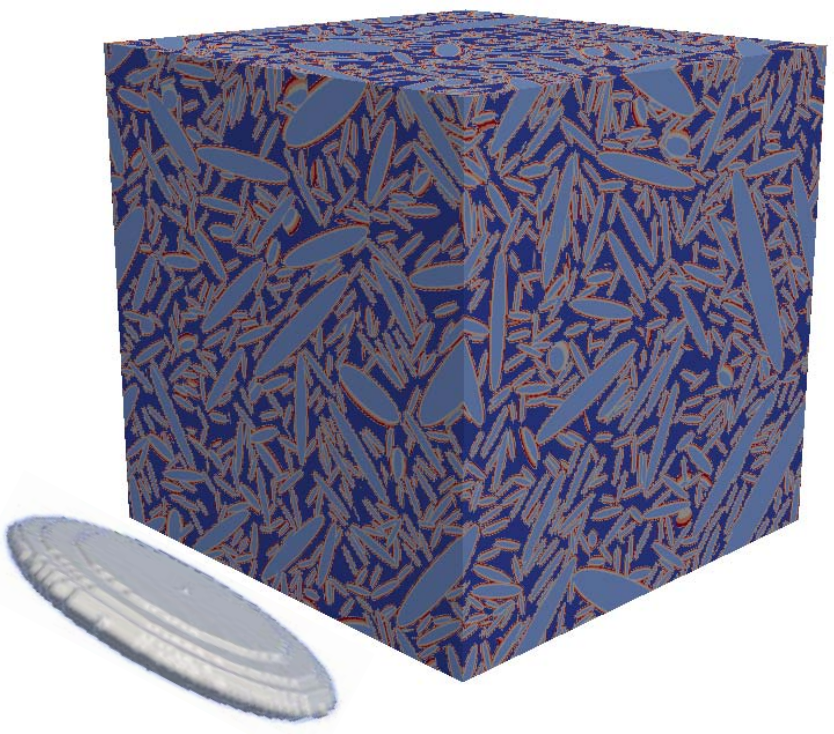

d) Oblate spheroidal aggregate particles $(a: b: c=2: 2: 0.25)$

Fig. 1 Randomly generated mesostructure containing $30 \%$ volume of spherical and non-spherical aggregate particles ranging from 0.15 to $2 \mathrm{~mm}$ (Fuller-Thompson gradation) in a $7.5 \times 7.5 \times 7.5 \mathrm{~mm}$ computational cube (voxel size $=16.7 \mu \mathrm{m})$.

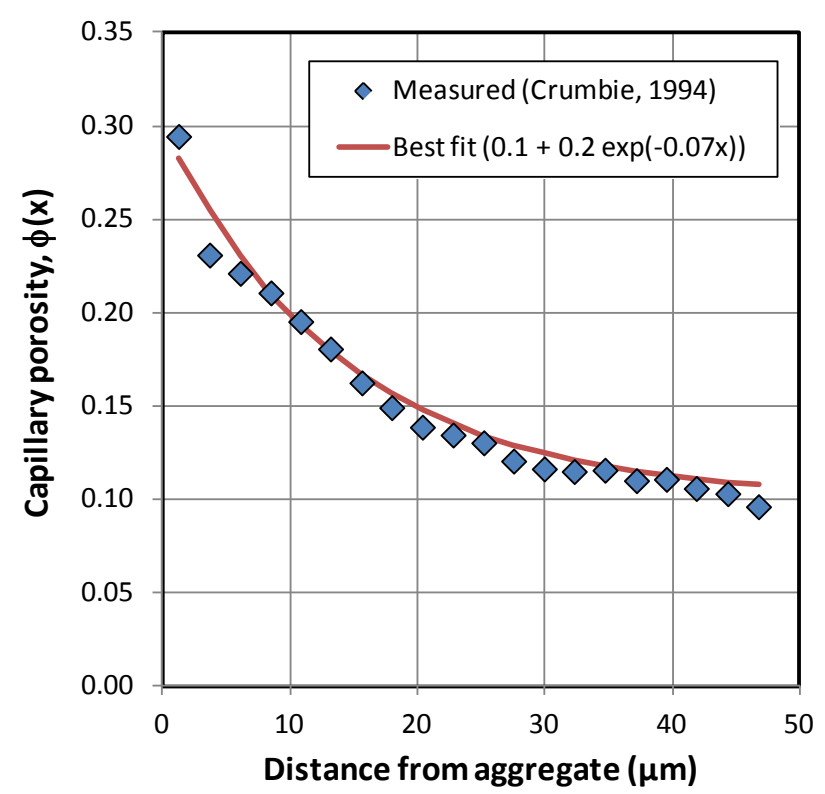

Fig. 2 Average porosity as a function of distance from the aggregate surface measured by Crumbie [15] for an OPC concrete with w/c $=0.4$ after 28 days of curing. Data is fitted using the exponential function shown in Eq. 2.

Eq. 2 can be solved by considering that the porosities of ITZ $\left(\phi_{I T Z}\right)$ and bulk cement paste $\left(\phi_{\text {bulk }}\right)$ are related to the total capillary porosity $(\phi)$ by the following equation:

$$
\phi=V_{I T Z} \phi_{I T Z}+V_{b u l k} \phi_{b u l k}
$$

Where $V_{\text {ITZ }}$ and $V_{\text {bulk }}$ are the volume fractions of the ITZ and bulk paste respectively, and $\phi$ can be estimated from the $\mathrm{w} / \mathrm{c}$ ratio and degree of hydration $(\alpha)$ based on the empirical model derived from the work of Powers and Brownyard [22]: 


$$
\phi=\frac{(w / c)-0.36 \alpha}{(w / c)+0.32}
$$

Once the distribution of porosity in the cement paste is known, the diffusivity of voxels in the ITZ and bulk paste can be estimated as a function of $x$, using the following equation derived from the work of Garboczi and Bentz [9]:

$$
\frac{D(x)}{D_{0}}=0.001+0.07 \phi(x)^{2}+1.8 H[\phi(x)-0.18] \times[\phi(x)-0.18]^{2}
$$

Where $D(x) / D_{0}$ is the ratio of diffusivity of the phase of interest to the free diffusivity $\left(D_{0}\right)$ and $H$ is the Heaviside function having a value of 1 when $\phi>0.18$ or a value of 0 otherwise. This equation was established by fitting experimental results of cement pastes at a range of $w / c$ ratios $(0.4$ to 0.6$)$, and degrees of hydration (0.6 to 0.9). The constant term (0.001) represents the limiting value of diffusion through $\mathrm{C}-\mathrm{S}-\mathrm{H}$ gel pores when the paste contains no capillary porosity, the $H$ term represents diffusion through percolated capillary porosity, and the second term is a fitting term that connects the two limiting behaviours. This equation assumes that diffusion occurs mainly through capillary pores as long as they remain percolated. When the capillary pores become depercolated, transport is then dominated by the gel pores within the C-S$H$. The percolation threshold is taken to be independent of w/c ratio and occurs at $\phi=0.18$ [9].

It should be noted that there are several versions of Eq. 5, each having slight variations in the fitting coefficients [Bentz et al., 2000]. However, we have applied the form presented in Eq. 5 as this is most commonly used and more importantly, it has been shown to give good agreement with diffusivity and conductivity [Halamickova et al., 1995; Garboczi \& Bentz, 1996] when coupled with the porosity equation from Young and Hansen [1987]:

$$
\phi=1-\frac{1+1.31 \alpha}{1+3.2(w / c)}
$$

Where the coefficient 3.2 represents the assumed specific gravity of cement and 1.31 represents the relative increase in solids volume due to the hydration. However, this equation considers only $C_{3} S$ reaction and does not properly account for chemical shrinkage.

\subsection{Simulation of diffusion process}

Fick's first law of diffusion coupled with mass conservation is then applied to simulate bulk diffusion through the mesostructure. At steady state and ignoring chemical interactions:

$$
\frac{\partial C}{\partial t}=\nabla \cdot(D \nabla C)=0
$$

Where $C$ is the concentration of the diffusing species and $t$ is time. It should be noted that in the finite difference scheme, transport can only occur between two voxels that share a face (Fig. 3). Voxels that share a vertex or an edge are assumed disconnected in the model. If two particular face-sharing voxels are of the same phase, then the diffusivity of the element connecting them is the diffusivity of that particular phase. If the face-sharing voxels are of different phases, then the diffusivity of the element connecting them is approximated using a simple series combination. Taking a trivial case of a mesostructure that consist of either ITZ, bulk paste or aggregate voxels as an example, the resulting network will contain three types of elements with the following diffusivities: $D_{I T Z}, D_{\text {bulk }}$ or $D_{\text {ITZ-bulk, }}$, where the latter is the diffusivity of an element connecting ITZ to a bulk paste voxel (or vice-versa) given by:

$$
\frac{2}{D_{I T Z-b u l k}}=\frac{1}{D_{I T Z}}+\frac{1}{D_{\text {bulk }}}
$$

However, if the ITZ is further divided into several layers each having its own porosity and transport property as a function of $x$ defined in Eq. 2 and Eq. 6, then the resulting network will contain many more types of elements, but similar rules will apply when determining their connectivity and diffusivity as described earlier. 


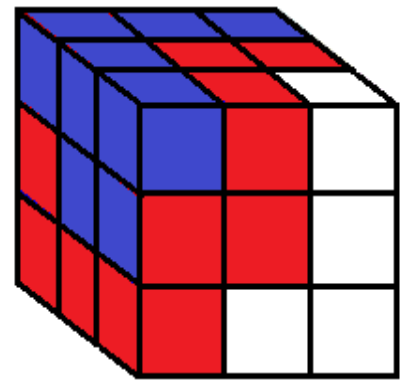

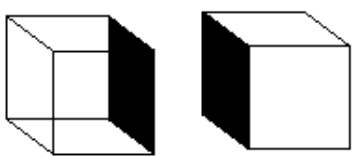

(Connected)
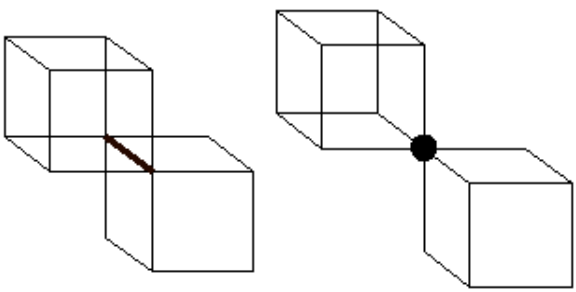

(Not connected)

Fig. 3 Schematic showing the connectivity of the voxelised mesostructure. Transport occurs between voxels with a shared face only.

A constant concentration boundary condition is applied for all the nodes on the inlet and outlet surfaces. This leads to a set of algebraic simultaneous equations which can be solved by an iterative algorithm. Fig. 4 shows an example of the concentration distribution for a sample containing spherical aggregate particles at steady-state conditions. Once the concentration in all the nodes is obtained, the effective diffusivity of the media $D$ can be calculated from:

$$
D=\frac{Q L}{A \Delta C}
$$

Where $Q$ is the sum of the flow through all elements on the outlet surface at steady-state, $A$ is the cross section area and $L$ is the length over which the concentration gradient $\Delta C$ is imposed.

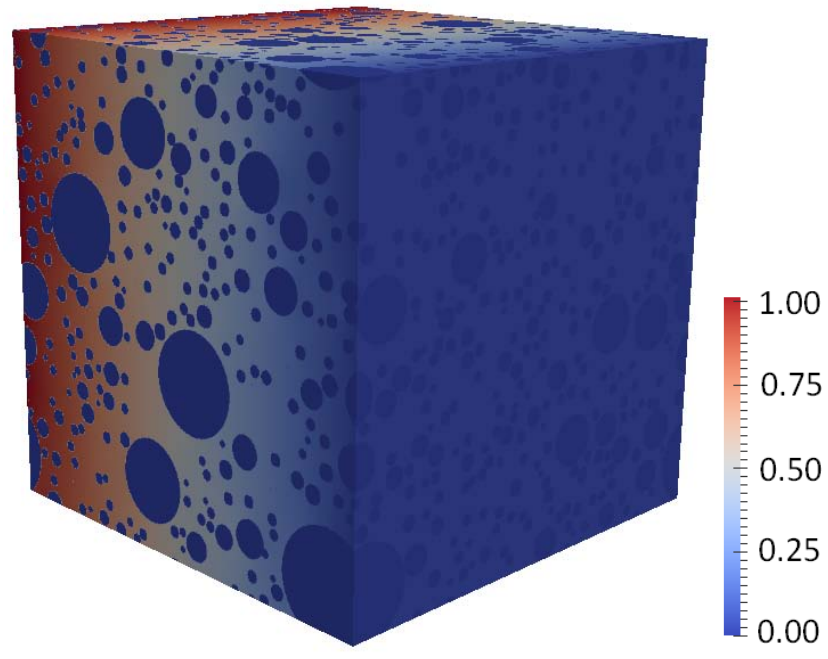

Fig. 4 Concentration distribution for sample containing $30 \%$ volume spherical aggregate particles, $0.4 \mathrm{w} / \mathrm{c}$ ratio and $80 \%$ degree of hydration, at steady-state condition.

\section{Representative elementary volume}

\subsection{Size and statistical effects}

Our numerical model is inevitably subjected to effects of digital resolution, finite sample size and statistical fluctuation. Size effects arise because the physical volume of the numerical sample has been limited to speed up computation, while statistical effects occur because of the random nature of the model. For example, the placement of aggregate particles is determined from randomly generated numbers, therefore the created mesostructure would be different at each realisation. Thus, it is important to determine the requirements in terms of resolution, sample size and number of realisations to obtain representative results.

In order to evaluate the size and statistical effects, we predicted the diffusivity ratio $D / D_{c p}$ of a sample at 0.5 $\mathrm{w} / \mathrm{c}$ ratio, $80 \%$ degree of hydration and containing $40 \%$ vol. aggregate particles ( 0.15 to $1 \mathrm{~mm}$ ) at numerical sample sizes of $2.5,5.0$ and $7.5 \mathrm{~mm}$. Thus, the ratio of sample size to the size of largest aggregate particle ranges from 2.5 to 7.5 . Note that $D_{c p}$ is the effective diffusivity of a neat cement paste at equivalent w/c ratio and degree of hydration. At least four realisations were conducted for each sample size. 
The results are plotted in Fig. 5 . As expected, each realisation produces a slightly different result and the precision improves with increase in sample size. Incorporating an ITZ in the model increases scatter slightly due to additional heterogeneity, but the overall variability is insignificant (to the third decimal point) even for the smallest sample size. By averaging over several realisations (shown as dotted lines in Fig. 5), the $D / D_{c p}$ ratios differ by no more than $0.5 \%$. This shows that a representative result can be obtained by averaging several realisations on a sample size that is at least 2.5 times the largest aggregate size. In all the simulations hereinafter, this criterion will be satisfied. It should be recalled that the required computational time and memory grows with the cube of the sample size. So roughly speaking, we can perform 27 simulations of the $2.5 \mathrm{~mm}$ sample for each simulation of the $7.5 \mathrm{~mm}$ sample. Furthermore, and more importantly, one has to use the smallest sample size when memory is limited.

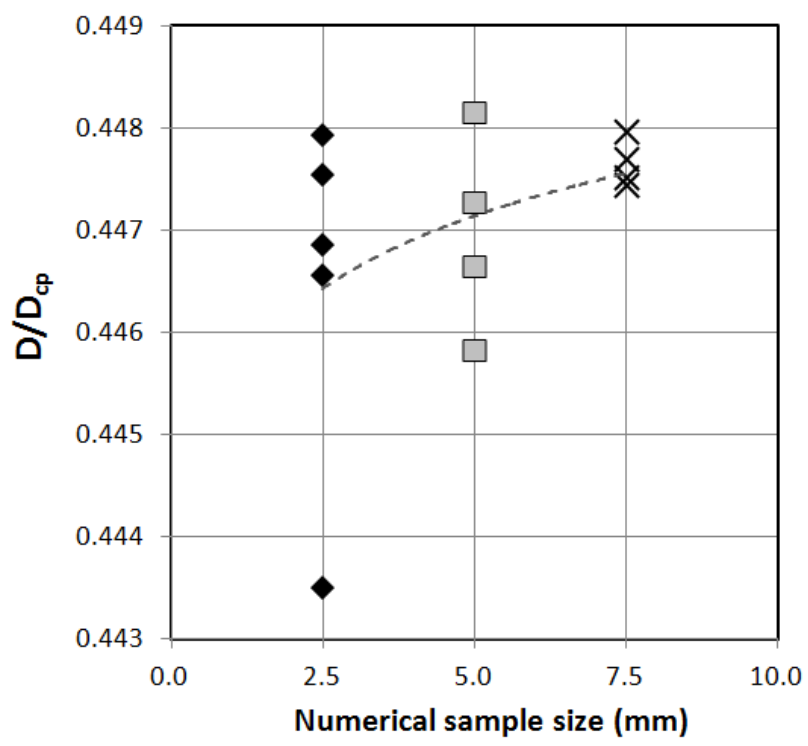

(a) No ITZ

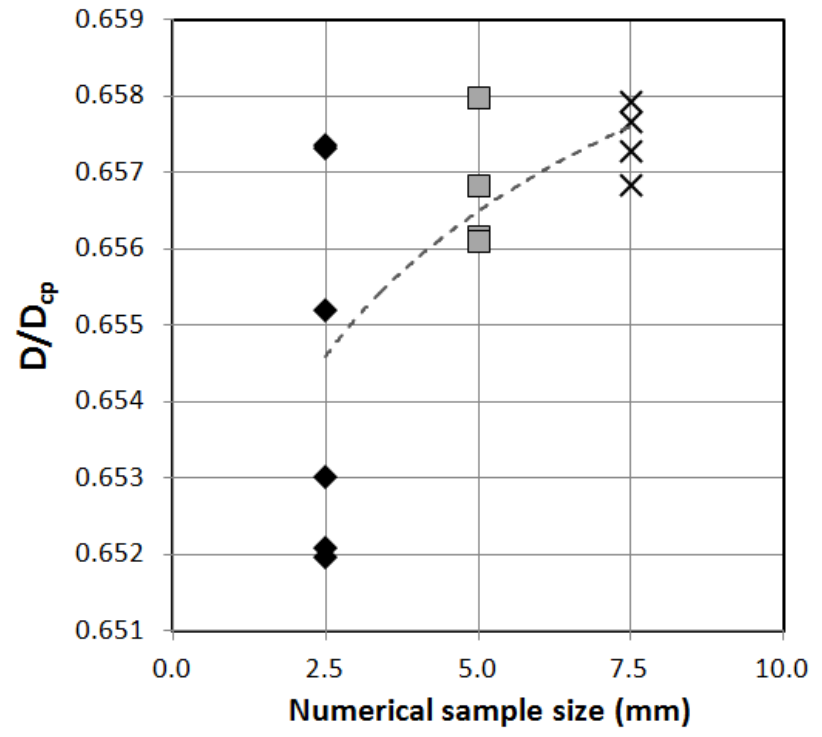

(b) ITZ = $50 \mu \mathrm{m}$ wide

Fig. 5 Effect of sample size and statistical fluctuations on the diffusivity ratio $D / D_{c p}$ for a mesostructure at $0.5 \mathrm{w} / \mathrm{c}$ ratio and $80 \%$ degree of hydration, containing $40 \%$ vol. aggregate particles ( 0.15 to $1 \mathrm{~mm}$, Fuller-Thompson). Simulations were carried out at $25 \mu \mathrm{m}$ resolution, with and without ITZ.

\subsection{Required resolution to capture the effect of aggregate particles and ITZ}

The presence of impermeable aggregate particles in the mesostructure exerts two opposite effects on mass transport. It reduces transport by decreasing the volume of cement paste (dilution) and increasing the length of the flow paths (tortuosity), but the formation of porous ITZ and its potential percolation may drastically increase transport. The ability of our model to capture these effects accurately, like any other model that uses a digitised microstructure as an input, should increase with resolution. However, the available computational resource imposes a practical limit on the resolution to be used.

Table 2 shows the effect of resolution on the diffusivity ratio $D / D_{c p}$ for a mesostructure containing $50 \%$ sand volume fraction for two different aggregate shapes. The simulations were carried out at voxel sizes between $12.5 \mu \mathrm{m}$ and $50 \mu \mathrm{m}$. As expected, the predicted transport property increases when smaller voxels are used because the aggregate shape and the connectivity between the ITZ voxels are better represented. Our results suggest that using a voxel size of $16.7 \mu \mathrm{m}$ seems to be a good compromise for achieving good accuracy in a reasonable computational time. Therefore, resolution of at most $16.67 \mu \mathrm{m}$ is chosen in the following simulations. On a single 64-bit PC (Processor: 3.4 GHZ, RAM memory: $8 \mathrm{~GB}$ ), the simulation takes a few hours to converge depending on the aggregate volume fraction.

Table 2 Effect of resolution on the diffusivity ratio $D I D_{c p}$ for samples containing spherical and tri-axial ellipsoidal aggregate particles (2: 1: 0.5 ) at different w/c ratios. The samples contain $50 \%$ sand volume fraction $\mathrm{V}_{\mathrm{a}}(0.15-2 \mathrm{~mm}$, Fuller). Simulations were carried out at $80 \%$ degree of hydration and assuming a $50 \mu \mathrm{m}$ ITZ width. 


\begin{tabular}{cccccc}
\hline \multirow{2}{*}{ Aggregate shape } & \multirow{2}{*}{$w / \mathrm{c}$} & \multicolumn{4}{c}{ Resolution $(\mu \mathrm{m})$} \\
\cline { 3 - 6 } & & 50 & 25 & 16.67 & 12.5 \\
\hline \multirow{2}{*}{ Spherical } & 0.4 & 0.382 & 0.452 & 0.469 & 0.486 \\
$(1: 1: 1)$ & 0.5 & 0.400 & 0.528 & 0.563 & 0.586 \\
& 0.6 & 0.318 & 0.380 & 0.396 & 0.410 \\
\hline \multirow{2}{*}{ Tri-axial ellipsoidal } & 0.4 & 0.324 & 0.396 & 0.424 & 0.438 \\
(2: $1: 0.5)$ & 0.5 & 0.354 & 0.483 & 0.531 & 0.553 \\
& 0.6 & 0.268 & 0.334 & 0.361 & 0.373 \\
\hline
\end{tabular}

\section{Results}

\subsection{Comparison with experimental results}

The predicted diffusivities were compared with two sets of experimental results. The first set of data from Delagrave et al. [26] consists of mortars made with ASTM Type I cement at $0.45 \mathrm{w} / \mathrm{c}$ ratio and $0.15-0.6 \mathrm{~mm}$ crushed siliceous sand at $0 \%, 30 \%$ and $50 \%$ vol. fractions. $15 \mathrm{~mm}$ thick samples were cured in saturated lime solution for 3 months and then vacuum saturated in deionised water and tested using a migration cell technique similar to the one described by Buenfeld and El-Belbol [27]. The upstream compartment of the migration cell was filled with $0.5 \mathrm{M} \mathrm{NaCl}$ in $0.3 \mathrm{M} \mathrm{NaOH}$ solution, and a $10 \mathrm{~V}$ potential was applied across the cell. The chloride concentration in the downstream compartment was monitored for 3 weeks and the migration coefficient was obtained at steady-state according to the Nernst-Plank equation.

The second set of experimental data is from Wong et al. [28] who tested mortars made of CEM I at $0.30 \mathrm{w} / \mathrm{c}$ ratio and Thames valley sand $(<5 \mathrm{~mm})$ complying with BS 882 medium grading. The sand volume fraction ranged from 0 to $65 \%$. The samples were sealed cured for 3 days and then preconditioned by drying at gradually increasing temperature up to $50^{\circ} \mathrm{C}$ to constant weight over a period of 90 days. Oxygen diffusivity was determined by exposing the opposite faces of the sample to a stream of pure oxygen and nitrogen at equal pressure. The gases counter diffuse through the sample and the oxygen concentration at the outlet face was monitored using a zirconia analyser until steady-state condition. The intrinsic diffusivity (average of three replicates) was then determined using Fick's first law of diffusion.

The degree of hydration is required in order to simulate these data. For the mortars tested by Delagrave et al. [26], a degree of hydration of 0.84 will be assumed based on the curing regime and age of the samples at the time of testing. This value is consistent with that used by Bentz et al. [29] and Kamali-Bernard et al. [4]. These authors have attempted to simulate the data of Delagrave et al [26] by creating an initial microstructure using the mineralogical composition of the cement and then simulating the hydration of the initial microstructure for 1000 cycles. For the mortars tested by Wong et al. [28], the degree of hydration was estimated to be 0.56 based on the non-evaporable water content measured by loss-on-ignition on companion cement pastes.

Fig. 6 compares our numerical simulations with the experimental results, which are plotted against aggregate volume fraction. Note that the actual aggregate size distribution from the original reference was an input to our model and aggregate particles are assumed spherical. The simulations were carried out at ITZ widths of $0 \mu \mathrm{m}, 25 \mu \mathrm{m}$ and $50 \mu \mathrm{m}$. As expected, the diffusivity ratio $D / D_{c p}$ decreases with an increase in aggregate volume fraction. The simulations also show that $D / D_{c p}$ increases with an increase in ITZ width. A good agreement between experimental data and simulations is observed when the ITZ is between 25 and $50 \mu \mathrm{m}$. 


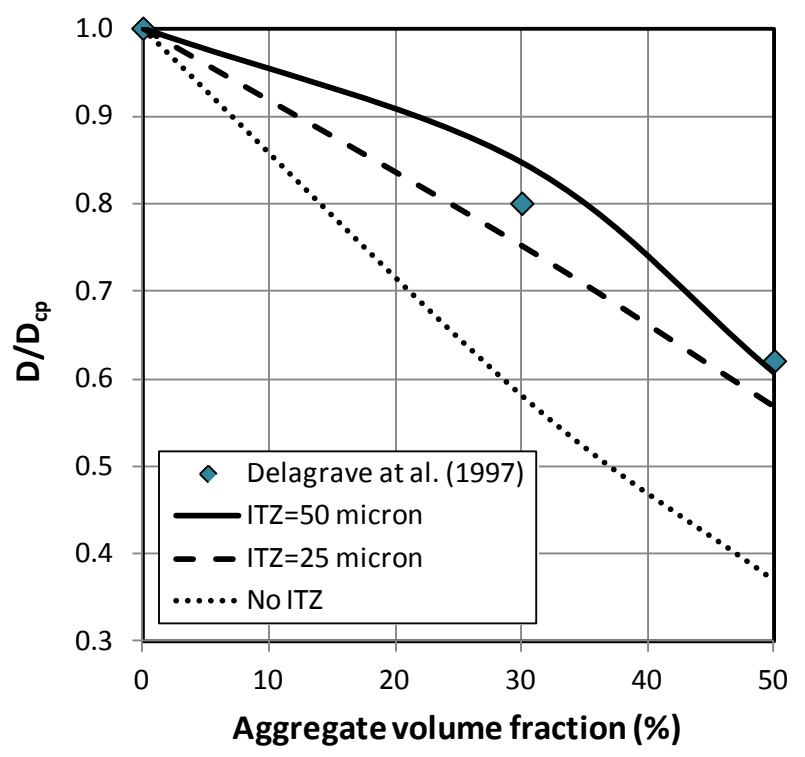

(a) Delagrave et al. [26]

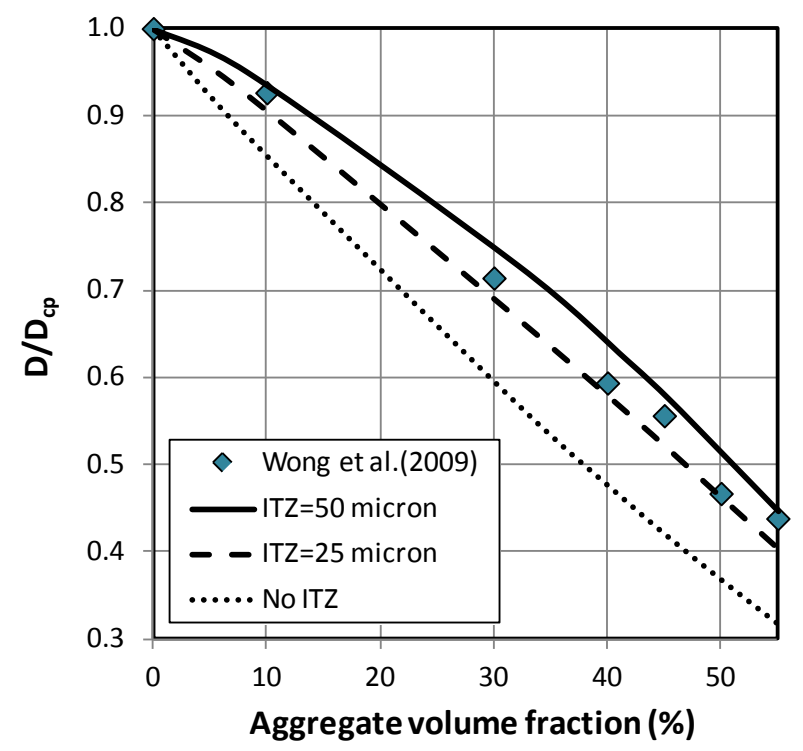

(b) Wong et al. [28]

Fig. 6 Comparison between the numerical simulations and experimental results of Delagrave et al. [26] and Wong et al. [28]

\subsection{Sensitivity analysis}

Sensitivity analyses were performed using the proposed model to examine the relative influence of $w / c$ ratio, degree of hydration, aggregate volume fraction and maximum aggregate size on diffusivity.

Fig. 7 a shows the effect of $w / c$ ratio on the diffusivity ratio $\left(D / D_{0}\right)$ of samples at a constant degree of hydration of 0.8 . Fig. $7 \mathrm{~b}$ shows the effect of degree of hydration on the diffusivity ratio $\left(D / D_{0}\right)$ of samples at a constant $\mathrm{w} / \mathrm{c}$ ratio of 0.5 . Both simulations were carried out for $0-50 \%$ volume fraction of spherical aggregate

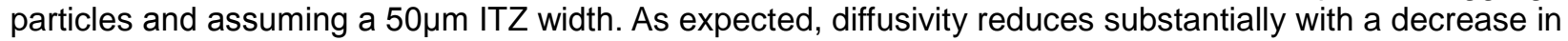
$\mathrm{w} / \mathrm{c}$ ratio. At equal $\mathrm{w} / \mathrm{c}$ ratio and aggregate content, diffusivity decreases with an increase in degree of hydration (increase in curing age). Diffusivity also reduces significantly with an increase in aggregate content. However, it was not computationally and practically feasible to pack more than $55 \%$ volume fraction of aggregate at the particle size distribution used in this study.

Fig. 8 shows the effect of maximum aggregate size on the diffusivity ratio $D / D_{c p}$ at $w / c$ ratios of $0.4,0.5$ and 0.6 , for aggregate size distributions of $0.15-0.5 \mathrm{~mm}, 0.15-1.0 \mathrm{~mm}, 0.15-2.0 \mathrm{~mm}$ and $0.15-4.0 \mathrm{~mm}$. The simulations were carried out for $0-50 \%$ aggregate volume fraction, $80 \%$ degree of hydration and assuming an ITZ width of $50 \mu \mathrm{m}$. The results show that decreasing the size of aggregate particles has a slight tendency to increase diffusivity. This can be attributed to the fact that more ITZ is produced when smaller aggregate particles are used. However, the magnitude of this effect depends on the contrast in diffusivity between the ITZ and bulk cement paste $\left(D_{I T Z} / D_{\text {bulk }}\right)$, which in turn is related to their respective porosities.

For example, the diffusivity contrast $D_{1 T Z} / D_{\text {bulk }}$ for samples containing $0.15-4.0 \mathrm{~mm}$ aggregate particles predicted in Fig. 8 range from 2.3 to 2.7 for w/c 0.4 , from 4.2 to 4.8 for w/c 0.5 , and from 2.2 to 2.4 for w/c 0.6 . Since the $D_{I T Z} / D_{\text {bulk }}$ for w/c 0.5 is significantly higher, the effect of changing aggregate size on diffusivity is also more pronounced compared to w/c 0.4 or 0.6 , as shown in Fig. 8. This also explains the observation that $D / D_{c p}$ is highest for w/c 0.5 , followed by w/c 0.4 and 0.6 .

This seemingly peculiar behaviour can be understood by examining the nature of Eq. 6 that relates $D / D_{o}$ to porosity and how this in turn influences the diffusivity contrast $D_{I T Z} / D_{\text {bulk. }}$ This equation is plotted in Fig. 9. Following the approach of Garboczi \& Bentz [1,3], these diffusivity values are averaged in two subsets, those lying within the ITZ and those in the bulk paste to give two values $D_{\text {ITZ }}$ (shown in solid arrows) and $D_{\text {bulk }}$ (dotted arrows). The resulting $D_{\text {ITZ }} / D_{\text {bulk }}$ values for different w/c ratios are shown. This comparison shows that the w/c 0.5 sample has porosity values that lie in the range that gives the highest contrast in $D_{\text {ITZ }} / D_{\text {bulk }}$. Because of the interactive feature of our model, $D_{1 T Z} / D_{\text {bulk }}$ will also change depending on the degree of hydration and the assumed ITZ width. Nevertheless, the range of $D_{I T Z} / D_{\text {bulk }}$ values reported here are consistent with those from other studies $[2,4,6]$. 
It can be concluded that the aggregate volume fraction, water to cement ratio and degree of hydration are the most significant parameters affecting diffusivity. This finding agrees well with the reported results in the literature $[3,6]$.

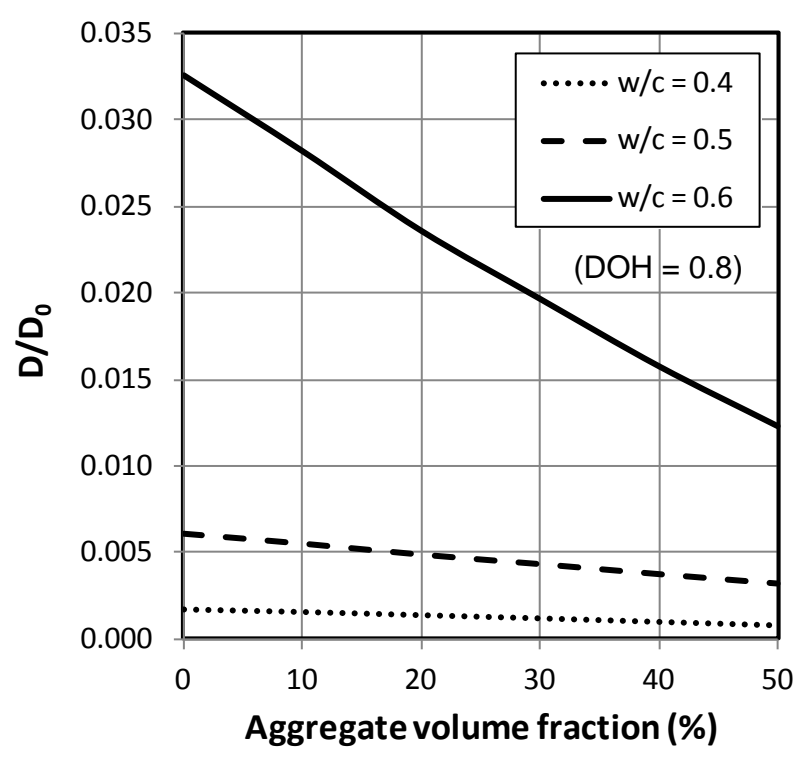

(a)

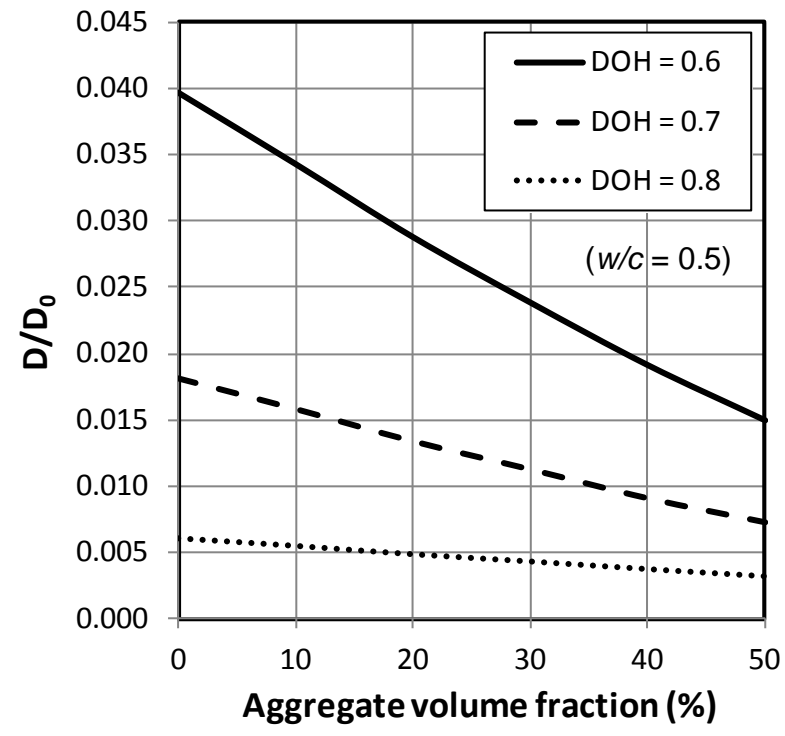

(b)

Fig. 7 Sensitivity analyses on the effect of a) w/c ratio and b) degree of hydration on $D / D_{o}$, assuming an ITZ width of $50 \mu \mathrm{m}$ and aggregate gradation of $0.15-2 \mathrm{~mm}$ (Fuller).

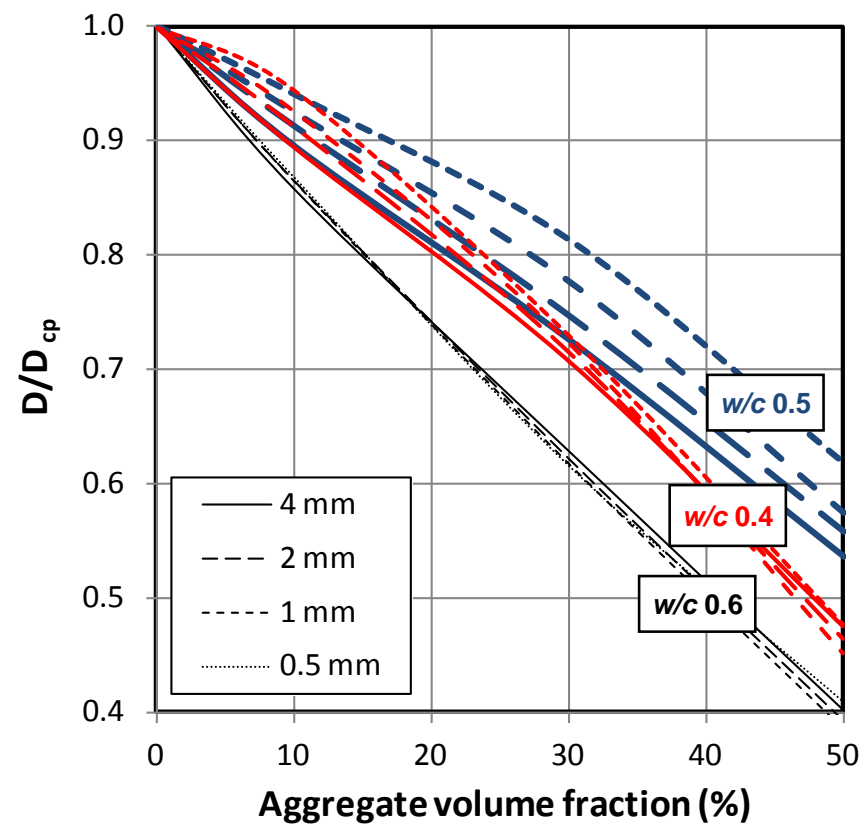

Fig. 8 Effect of maximum aggregate size on the $D / D_{c p}$ at w/c ratios of $0.4,0.5$ and 0.6 , assuming an ITZ width of $50 \mu \mathrm{m}$ and $80 \%$ degree of hydration. 


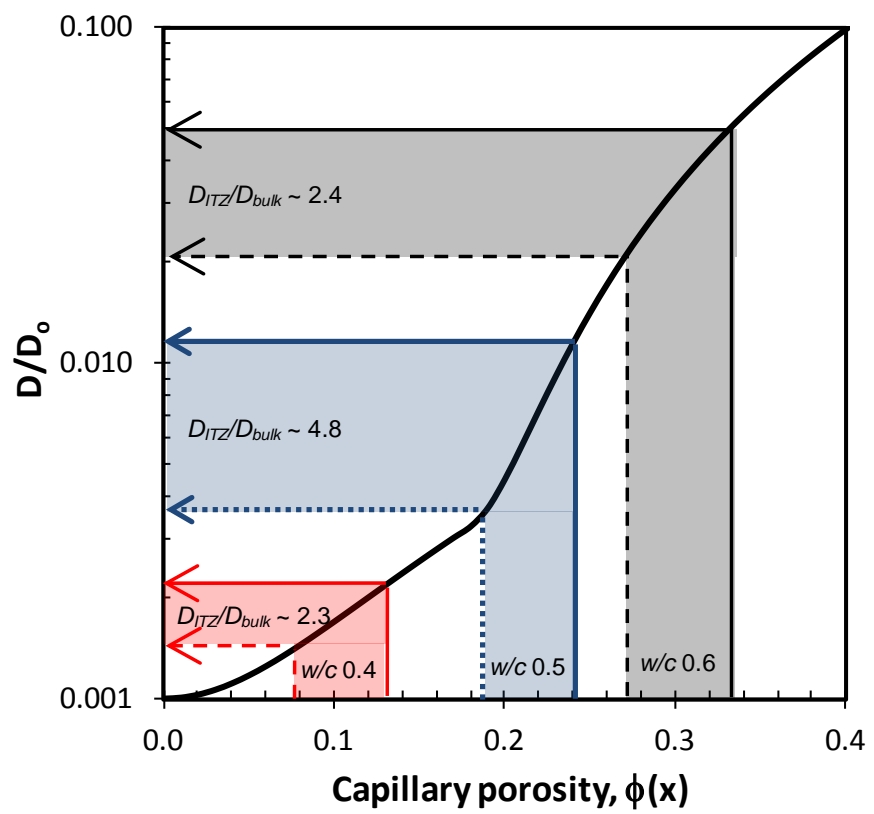

Fig. 9 Determination of $D_{I T Z} I D_{\text {bulk }}$ from the plot of $D / D_{o}$ against capillary porosity (Eq. 6). Values shown are for samples containing $50 \%$ vol. aggregate assuming an ITZ width of $50 \mu \mathrm{m}$ and $\mathbf{8 0} \%$ degree of hydration.

\subsection{Effect of aggregate shape}

Fig. 10 shows the effect of aggregate shape on the diffusivity ratio $D / D_{c p}$. Seven particle shapes were examined (Table 1). The aspect ratios of these were chosen to maintain a constant particle volume so that for a sample containing a certain volume fraction of aggregate particles, the total number of aggregate particles will not be influenced by a change in aggregate shape. Simulations were carried for w/c ratios of 0.4 and 0.5 , at $80 \%$ degree of hydration and assuming a $50 \mu \mathrm{m}$ ITZ width. Simulations were also carried out for the case of no ITZ. This allows us to isolate the effect of ITZ from the effect of reduction in the paste fraction (dilution) and the effect of tortuosity caused by the aggregate particles.

The simulations show that for all cases, diffusivity decreased when spherical aggregate particles are replaced with ellipsoidal ones. However, the effect is more pronounced for oblate spheroidal and tri-axial ellipsoidal compared to prolate spheroidal aggregate particles. The reduction in diffusivity is also more significant at higher aggregate fractions and at higher aspect ratio (more elongation) of the aggregate particles.

Several competing factors that influence transport properties occur when spherical aggregate particles are replaced with ellipsoidal ones. Because of the larger surface area of ellipsoidal aggregate particles, the amount of ITZ increases. This should enhance the percolation of ITZ [30] and therefore increase diffusivity. However, this is counter-balanced by a denser bulk cement paste (lower w/c ratio) as a result of water conservation in the microstructure. In addition, the effective transport path length, that is the tortuosity effect, is increased with ellipsoidal aggregate particles. From the simulations, it is evident that the tortuosity effect is more significant compared to that of the ITZ. 


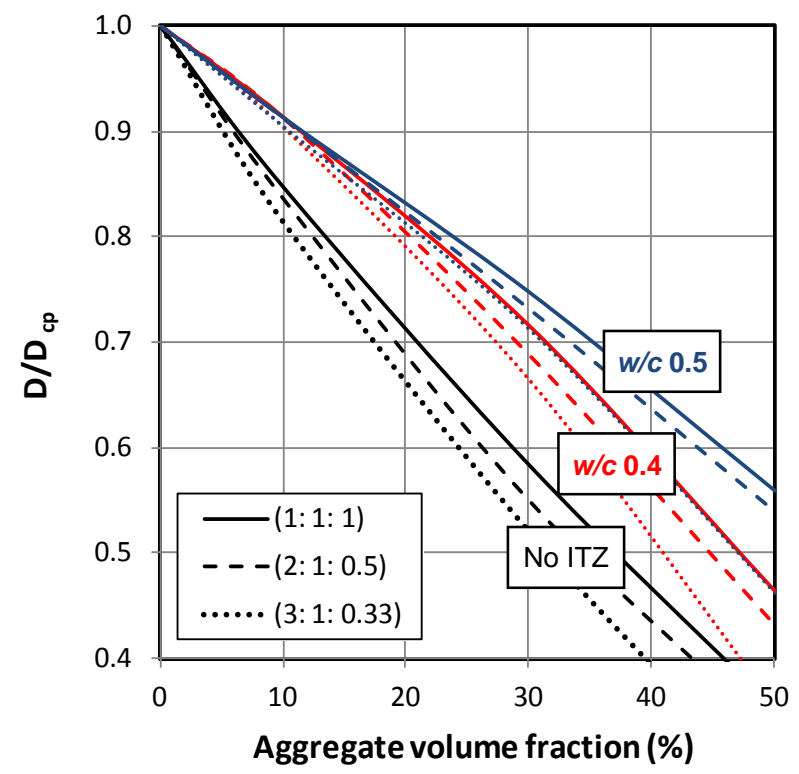

a) Tri-axial ellipsoidal $(a>b>c)$

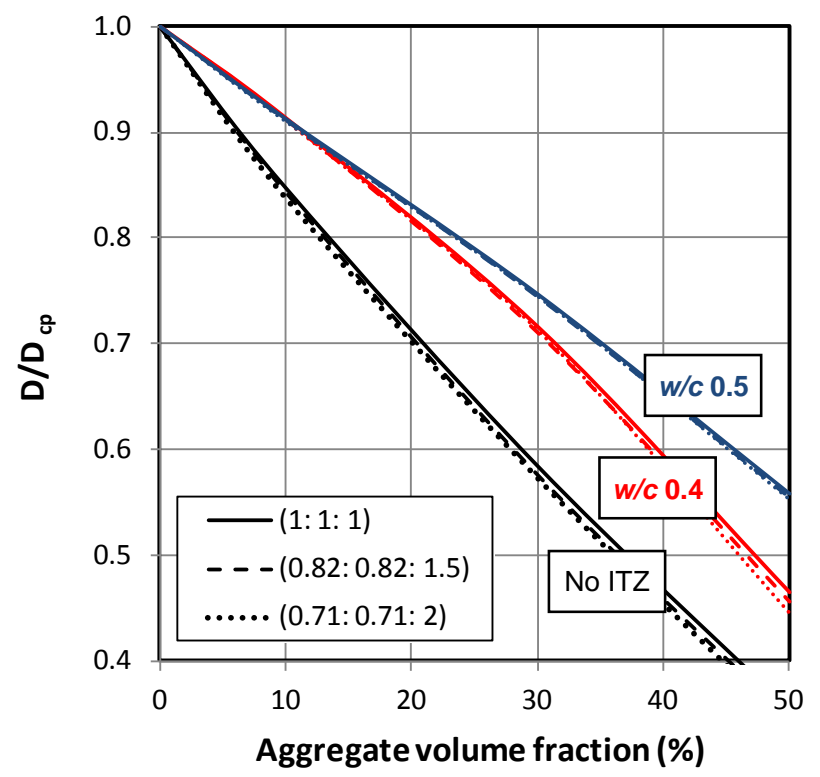

b) Prolate spheroidal $(\mathrm{a}=\mathrm{b}<\mathrm{c})$

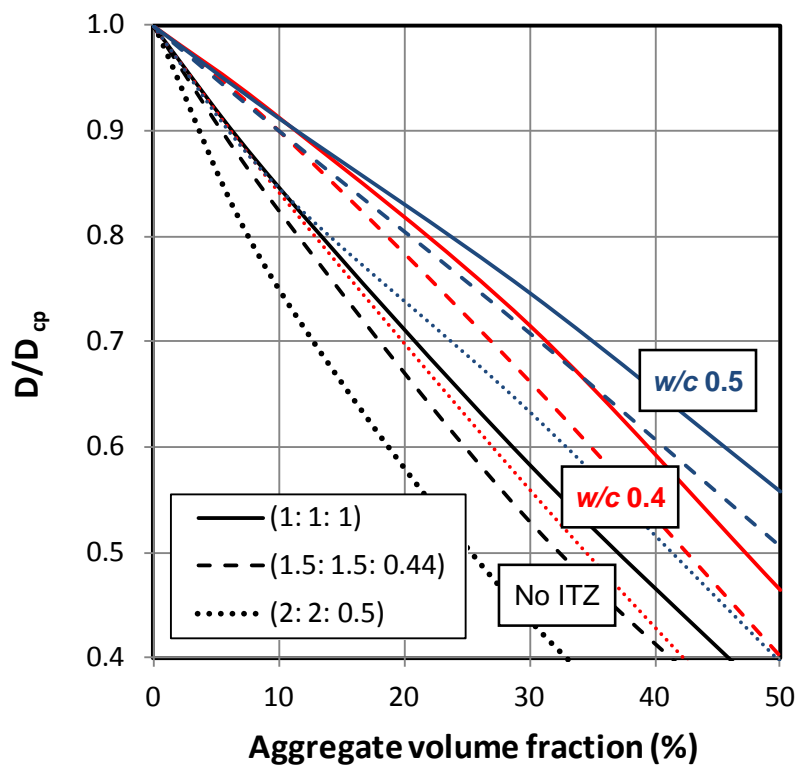

c) Oblate spheroidal $(a=b>c)$

Fig. 10 Effect of aggregate shape on $D / D_{c p}$ for w/c ratios of 0.4 and 0.5 , assuming an ITZ width of $50 \mu \mathrm{m}, 80 \%$ degree of hydration and $\mathbf{0 . 1 5 - 2} \mathrm{mm}$ aggregate size. Simulations were also carried out for the case of no ITZ.

\subsection{Specific surface area and tortuosity}

To further explore the effect of aggregate shape on diffusivity, we examined the relationship between specific surface area of the aggregate particles, tortuosity of the hardened cement paste and diffusivity. The specific surface area is the total surface area of aggregate divided by the aggregate volume in the sample, while the paste tortuosity $(\tau)$ can be defined as:

$$
\tau=\frac{1-V_{a}}{D / D_{c p}}
$$


Where $V_{a}$ is the aggregate volume fraction and $D / D_{c p}$ is the ratio of diffusivity of the composite (with no ITZ) to the diffusivity of an equivalent neat cement paste. It should be noted that this tortuosity is the result of the presence of aggregate particles (that acts as dense inclusions blocking the transport path) and does not take into account the inherent tortuosity of the pore structure. The tortuosity of the paste matrix for the case of spherical aggregate particles can also be calculated from the classical Maxwell's approximation (Eq. 11) and Bruggemann-Hanai equation (Eq. 12):

$$
\begin{aligned}
& \tau(\text { Maxwell })=1+0.5 V_{a} \\
& \tau(\text { Bruggeman }- \text { Hanai })=\left(1-V_{a}\right)^{-0.5}
\end{aligned}
$$

These equations are derived by considering the effective conductivity (diffusivity) of a composite containing spherical non-conductive particles embedded in a homogeneous matrix. The conductivity of the matrix is assumed to be constant as non-conductive particles are added, therefore capturing the effects of dilution and tortuosity [31, 32]. It can be shown that the tortuosity calculated using our results with Eq. 10 is in excellent agreement with Maxwell's equation (for the dilute region of $V_{a}<30 \%$ ) and Bruggemann-Hanai's equation. Errors were no more than 1.1\%, thus lending support to the use of Eq. 10 for estimating tortuosity of composites containing ellipsoidal aggregate particles.

Samples containing spherical aggregate particles have the lowest specific surface area $\left(\sim 13.7 \mathrm{~mm}^{-1}\right)$. This is followed by samples with prolate spheroidal (14.6-16.0 $\left.\mathrm{mm}^{-1}\right)$, tri-axial ellipsoidal $\left(17.4-23.3 \mathrm{~mm}^{-1}\right)$ and oblate spheroidal aggregate particles $\left(18.3-29.0 \mathrm{~mm}^{-1}\right)$. It should be noted that the specific surface areas were calculated from the continuum representation of the aggregate particles and not from the digitised mesostructure since the surface area obtained by counting voxel faces incurs significant error [33]. Interestingly, the results show that there is a very strong linear correlation between specific surface area and paste tortuosity (Fig. 11a) and between specific surface and diffusivity (Fig, 11b). The correlation coefficient $\left(R^{2}\right)$ is better than 0.9 in all cases. This could be used as a means to estimate the effect of other aggregate types on diffusivity based on their specific surface area at a constant particle size distribution. The results show that replacing spherical with ellipsoidal aggregate particles increases the paste tortuosity, which results in a decrease in diffusivity. This is most significant for oblate spheroidal, followed by tri-axial ellipsoidal and prolate spheroidal aggregate particles.

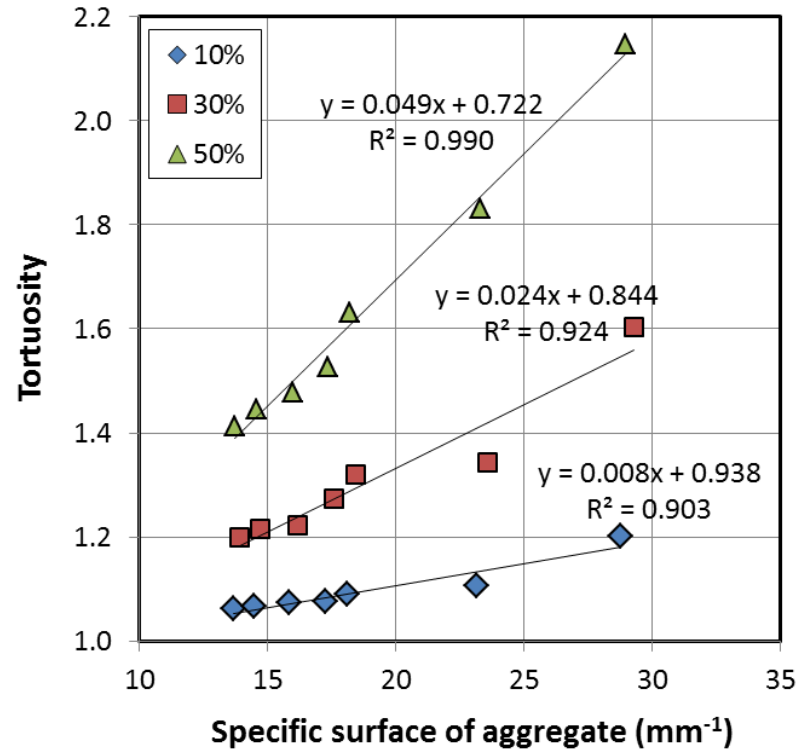

(a)

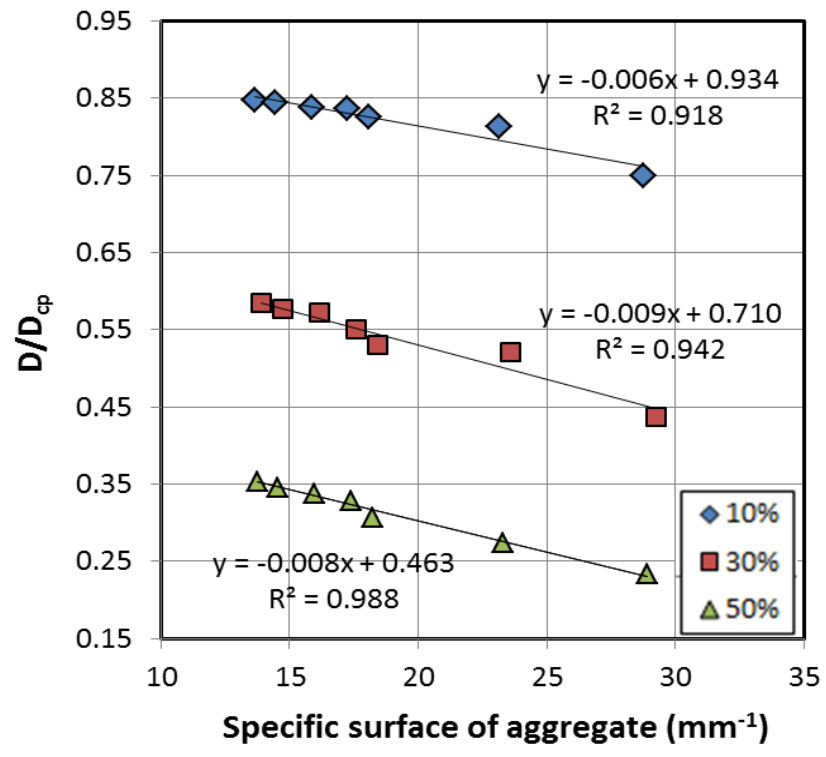

(b)

Fig. 11 Correlation between the specific surface area of aggregate particles, tortuosity of the cement paste and diffusivity ratio $D / D_{c p}$ for samples (no ITZ) containing $10 \%, 30 \%$ and $50 \%$ vol. aggregate simulated in Fig. 10. 


\subsection{Effect of aggregate orientation}

Since aggregate particles are placed randomly in the computational cube, the mesostructures tested in this study exhibit isotropic behaviour. The transport property measured along the three principal axes should be identical and can be represented as a single scalar value. To demonstrate this, we predicted the diffusivity of a $0.4 \mathrm{w} / \mathrm{c}$ ratio sample containing $30 \%$ volume fraction of randomly placed tri-axial ellipsoidal aggregate particles with aspect ratio 2:1:0.5. The values of $D / D_{c p}$ calculated along the $\mathrm{x}, \mathrm{y}$ and $\mathrm{z}$ axes (Fig. 12) are almost identical and equal to $0.695,0.687$ and 0.687 respectively. It should be noted that in practice, diffusivity could be influenced by the orientation of the aggregate particles. For example, Fig.13 shows the mesostructure of the same sample, but with the aggregate particles placed in a way such that the largest radius is in the $x$-direction and the smallest radius in the $y$-direction. Simulations of the transport property show that the diffusivity in the $y$-direction is now significantly smaller than the diffusivity in the $x$ - or zdirection.
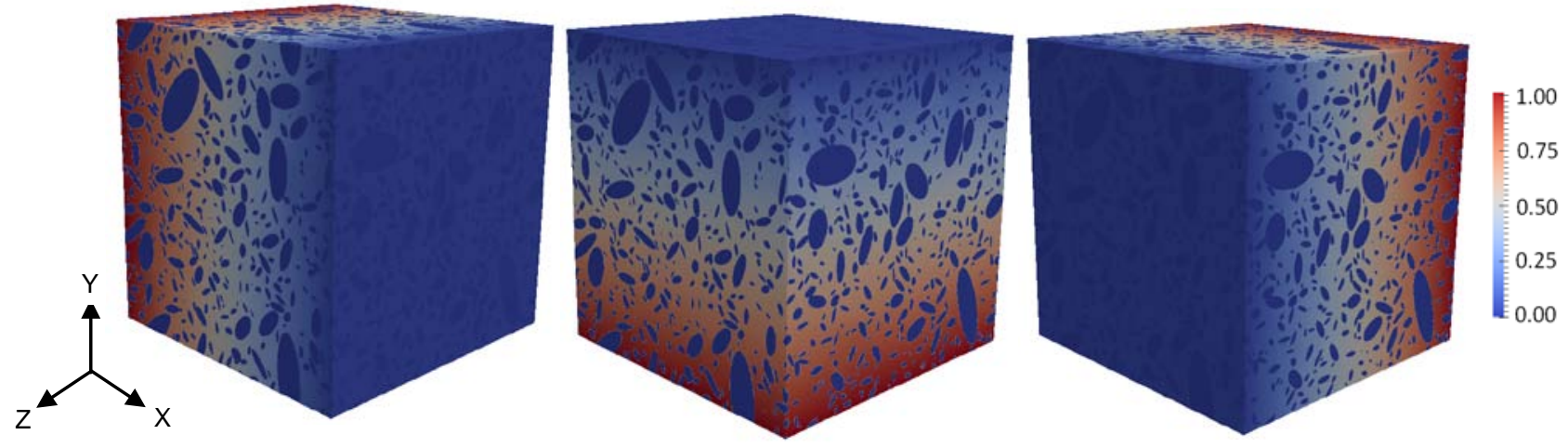

Fig. 12 Concentration distribution for sample containing $30 \%$ volume tri-axial ellipsoidal aggregate particles with aspect ratio (2:1:0.5), $0.4 \mathrm{w} / \mathrm{c}$ ratio and $80 \%$ degree of hydration, at steady-state condition.

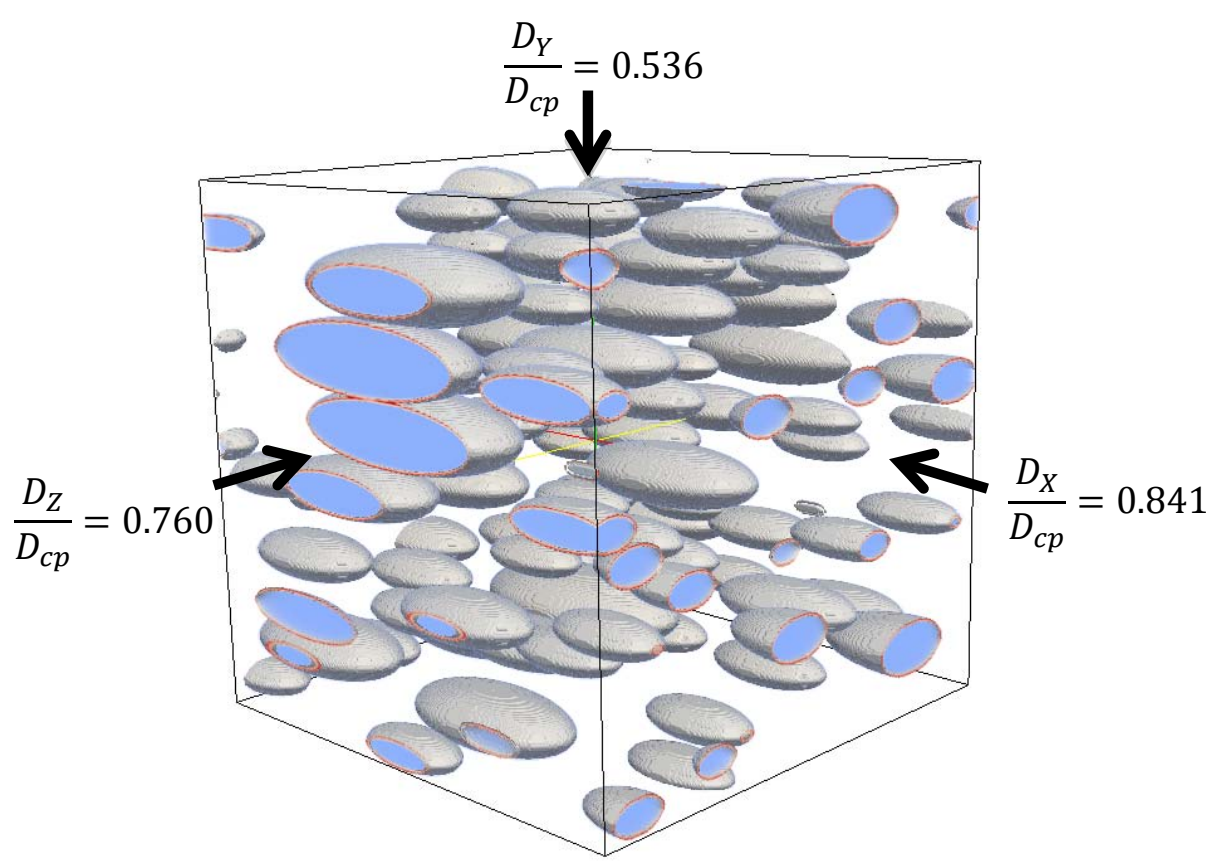

Fig. 13 Mesostructure containing 30\% volume tri-axial ellipsoidal aggregate particles with aspect ratio $(2: 1: 0.5)$ and aligned with the largest radius in the $x$-direction. The diffusivity shows significant anisotropy. For the sake of clarity, only aggregate particles larger than $1 \mathrm{~mm}$ are shown. 


\section{Discussion}

As mentioned previously, the presence of impermeable aggregate particles in concrete has different and opposite effects on diffusivity. Adding aggregate particles produces ITZ, but this is accompanied by a decrease in total porosity because cement paste is replaced by non-porous aggregate particles (dilution) and also an increase in the tortuosity of the cement paste. The dilution and tortuosity effects tend to decrease diffusivity. Considering that the $D / D_{o}$ for all cases is higher than the line representing dilution and tortuosity, one can conclude that the creation of ITZ has a positive effect on diffusivity. However, the magnitude of this is dependent on the diffusivity contrast between the ITZ and bulk cement paste, $D_{I T Z} / D_{\text {bulk}}$, and this in turn is dependent on their respective porosities. At constant aggregate content, increasing the ITZ width or using a finer aggregate size creates more ITZ, but this is accompanied by densification of bulk cement paste as a result of water conservation in the microstructure. Our simulations show that when the contrast $D_{\text {ITZ }} / D_{\text {bulk }}$ is $<$ 2.5 , these effects tend to cancel each other so that the net contribution of the ITZ on the overall diffusivity is insignificant.

In our simulations, the w/c 0.5 sample consistently has the highest $D_{I T Z} / D_{\text {bulk }}$ but this will change depending on the degree of hydration and assumed ITZ width. Some researchers have reported that the percolation of ITZ can significantly increase the transport properties of mortar and concrete [24, 34]. In the present study, a burning algorithm [35] based on the same rules for connectivity shown in Fig. 3 was used to determine the required aggregate content at which ITZ percolation occurs. It was found that the ITZ would form a percolated path in all of the examined samples when the aggregate volume fraction exceeds $30 \%$. However, this did not seem to produce a change in the trend of the predicted diffusivity, even for the w/c 0.5 sample which had the highest $D_{\text {ITZ }} / D_{\text {bulk }}$ contrast. The sensitivity analyses show that the most significant parameters influencing diffusivity were w/c ratio, degree of hydration and aggregate content, while the ITZ width, aggregate size and gradation have less influence on diffusivity. These findings agree well with Bentz et al. [3] \& Zheng et al. [6].

In practice, concrete has larger aggregate volume fraction and particle size range compared to the samples simulated in this study. Our modelling approach is applicable to concrete as long as the simulation is carried out on an appropriate numerical sample size and resolution. The main obstacle to achieving this is the huge computational resource required. Nevertheless, we can relate our current findings to concrete considering that the $D_{I T Z} / D_{\text {bulk }}$ contrast in concrete will not be significantly different from those examined in this study. We have shown that increasing aggregate size from 0.5 to $4 \mathrm{~mm}$ has little influence on the results. Furthermore, ITZ percolation has already occurred at 30\% aggregate volume fraction. Thus, we can reasonably expect that the findings from simulations carried out at larger aggregate size and volume fraction should not significantly deviate from those presented here.

The modelling approach described in this paper is particularly useful for evaluating the influence of various parameters on transport properties. Some of these effects are impossible to isolate and quantify by laboratory experimentation alone. Nevertheless, it should be kept in mind that the model uses several approximations in its development and is only strictly valid within these assumptions. The model contains simplification of the microstructure of concrete and empirical relationships that require calibration. In modelling transport, we assumed that all pores are accessible and contributing to transport. We also considered diffusion under steady-state conditions and ignored the effects of any chemical interactions.

Structures in service are often micro-cracked due to loading and drying shrinkage, but modelling microcracks and its effect on transport properties is challenging. The model described in this paper can readily be extended to simulate the effect of microcracks by introducing a fourth phase to represent them. For samples with high aggregate contents, these localised microcracks could become interconnected, thereby potentially compounding any effect of the ITZ. However, there are some experimental and theoretical studies that show that microcracking has very little effect on diffusion compared with pressure-induced flow [28, 36, 37]. This is because permeability is influenced by pore size, while diffusivity is more dependent on the total porosity, which is not significantly increased by the microcracks.

It should also be noted that the proposed model is not limited to mathematically or computationally manageable shapes. Arbitrary shapes can be incorporated into the models, for example by applying 3D shape statistics based on a collection of real particles [38]. Future work will concern the incorporation of real, random shapes into the model. Other phases such as air voids can also be included in the mesostructure model. The transport characteristics of air voids can be assigned depending on whether the air voids act as conductors or insulators [39]. 


\section{Conclusions}

This paper presented an investigation into the diffusivity of cement-based materials using a threedimensional interactive composite model containing spherical and ellipsoidal aggregate particles. Inputs to the model are the aggregate particle shape, size distribution and volume fraction, w/c ratio, degree of hydration and ITZ porosity gradient. The required numerical sample size and voxel resolution to capture the effects of dilution, tortuosity and ITZ were examined. It was found that representative results can be obtained by averaging several realisations on a sample size that is 2.5 times the largest aggregate particle, at $16.7 \mu \mathrm{m}$ voxel resolution. The validity of the model was verified by comparing with two sets of experimental results. The model was then applied to examine the influence of several parameters on diffusivity. The most significant parameters influencing diffusivity were w/c ratio, degree of hydration and aggregate content, while the ITZ width and aggregate size have less influence. The percolation of ITZ when aggregate volume fraction exceeds $30 \%$ did not result in an increase in diffusivity. The simulations also show that aggregate shape and orientation can have a significant effect on diffusivity. In all cases, diffusivity decreased when spherical aggregate particles were replaced with ellipsoidal aggregate particles due to an increase in the tortuosity of the cement paste. The effect is more pronounced for oblate spheroidal and tri-axial ellipsoidal compared to prolate spheroidal aggregate particles, and is also more significant at higher aggregate fractions and aspect ratios. There is a strong linear correlation between specific surface area of aggregate particles, paste tortuosity and the diffusivity at a constant particle size distribution for the range of aggregate particles investigated in this study. From the simulations, it is evident that the tortuosity and dilution effect is more significant compared to that of the ITZ. The modelling approach described in this paper is particularly useful for evaluating the influence of various parameters on transport properties, where laboratory experimentation alone would be difficult or impossible to isolate and quantify.

\section{Acknowledgments}

The research leading to these results has received funding from the European Union Seventh Framework Programme (FP7 / 2007-2013) under grant agreement 264448.

\section{References}

[1] E.J. Garboczi, D.P. Bentz, Advanced Cement Based Materials, 8 (1998) 77-88.

[2] B.H. Oh, S.Y. Jang, Cement and Concrete Research, 34 (2004) 463-480.

[3] D.P. Bentz, E.J. Garboczi, E.S. Lagergren, Cement Concrete and Aggregates, 20 (1998) 129-139.

[4] S. Kamali-Bernard, F. Bernard, W. Prince, Computational Materials Science, 45 (2009) 528-535.

[5] F. Bernard, S. Kamali-Bernard, Computational Materials Science, 61 (2012) 106-115.

[6] J.-J. Zheng, H.S. Wong, N.R. Buenfeld, Cement and Concrete Research, 39 (2009) 805-813.

[7] J.-J. Zheng, X.-Z. Zhou, Y.-F. Wu, X.-Y. Jin, Construction and Building Materials, 31 (2012) 151-156.

[8] L.-Y. Li, J. Xia, S.-S. Lin, Construction and Building Materials, 26 (2012) 295-301.

[9] E.J. Garboczi, D.P. Bentz, Journal of Materials Science, 27 (1992) 2083-2092.

[10] W.B. Fuller, S.E. Thompson, Trans. Am Soc Civ Eng, 59 (1907) 67-143.

[11] X. Jia, R.A. Williams, Powder Technology, 120 (2001) 175-186.

[12] M. Zalzale, P.J. McDonald, Cement and Concrete Research, 42 (2012) 1601-1610.

[13] M. Zhang, G. Ye, K.v. Breugel, Computational Materials Science, 68 (2013) 142-151.

[14] A. Nabovati, E.W. Llewellin, A.C.M. Sousa, Composites Part a-Applied Science and Manufacturing, 40 (2009) 860-869.

[15] A.K. Crumbie, in: Characterisation of the microstructure of concrete, PhD Thesis, Imperial College London, 1994.

[16] K.L. Scrivener, A.K. Crumbie, P. Laugesen, Interface Science, 12 (2004) 411-421.

[17] K.L. Scrivener, K.M. Nemati, Cement and Concrete Research, 26 (1996) 35-40.

[18] D.P. Bentz, E.J. Garboczi, ACI Materials Journal, 88 (1991) 518-529. 
[19] H.S. Wong, N.R. Buenfeld, Cement and Concrete Research, 36 (2006) 1091-1097.

[20] K.L. Scrivener, A.K. Crumbie, P.L. Pratt, Materials Research Society Symposium Proceedings, 114 (1988) 87-88.

[21] M.K. Head, H.S. Wong, N.R. Buenfeld, Cement and Concrete Research, 38 (2008) 1227-1231.

[22] T.C. Powers, T.L. Brownyard, J. Am. Concr. Inst. , 43 (1947).

[23] J.F. Young, W. Hansen, Materials Research Society Symposium Proceedings, 85 (1987) 313-322.

[24] P. Halamickova, R.J. Detwiler, D.P. Bentz, E.J. Garboczi, Cement and Concrete Research, 25 (1995) $790-802$.

[25] E.J. Garboczi, D.P. Bentz, Construction and Building Materials, 10 (1996) 293-300.

[26] A. Delagrave, J.P. Bigas, J.P. Ollivier, J. Marchand, M. Pigeon, Advanced Cement Based Materials, 5 (1997) 86-92.

[27] N.R. Buenfeld, S. El-Belbol, Magazine of Concrete Research, 43 (1991) 135-139.

[28] H.S. Wong, M. Zobel, N.R. Buenfeld, R.W. Zimmerman, Magazine of Concrete Research, 61 (2009) 571-589.

[29] D.P. Bentz, O.M. Jensen, A.M. Coats, F.P. Glasser, Cement and Concrete Research, 30 (2000) 953-962.

[30] D.P. Bentz, J.T.G. Hwang, C. Hagwood, E.J. Garboczi, K.A. Snyder, N. Buenfeld, K.L. Scrivener, Interfacial zone percolation in concrete: Effects of interfacial zone thickness and aggregate shape, in: S.M.S.G.F.P.R.L.W.S.J.P.W.

Diamond, Ld (Eds.) Microstructure of Cement-Based Systems/Bonding and Interfaces in Cementitious Materials, 1995, pp. 437-442.

[31] D.A.G. Bruggeman, Annalen der Physik, 416 (1935) 636-664.

[32] D.S. McLachlan, M. Blaszkiewicz, R.E. Newnham, Journal of the American Ceramic Society, 73 (1990) 21872203.

[33] E.J. Garboczi, D.P. Bentz, N.S. Martys, Methods in the Physics of Porous Media, 35 (1999) 1-41.

[34] D.N. Winslow, M.D. Cohen, D.P. Bentz, K.A. Snyder, E.J. Garboczi, Cement and Concrete Research, 24 (1994) 25-37.

[35] D.P. Bentz, E.J. Garboczi, Cement and Concrete Research, 21 (1991) 325-344.

[36] H.S. Wong, N.R. Buenfeld, J. Hill, A.W. Harris, Advances in Cement Research, 19 (2007) 35-46.

[37] B. Gérard, J. Marchand, Cement and Concrete Research, 30 (2000) 37-43.

[38] E.J. Garboczi, Cement and Concrete Research, 32 (2002) 1621-1638.

[39] H.S. Wong, A.M. Pappas, R.W. Zimmerman, N.R. Buenfeld, Cement and Concrete Research, 41 (2011) 10671077. 